\title{
Automated Termination Analysis for Logic Programs with Cut*
}

\author{
PETER SCHNEIDER-KAMP \\ Dept. of Mathematics and Computer Science, University of Southern Denmark, Denmark \\ JÜRGEN GIESL, THOMAS STRÖDER \\ LuFG Informatik 2, RWTH Aachen University, Germany \\ ALEXANDER SEREBRENIK \\ Dept. of Mathematics and Computer Science, TU Eindhoven, The Netherlands \\ RENÉ THIEMANN \\ Institute of Computer Science, University of Innsbruck, Austria \\ submitted 1 January 2003; revised 1 January 2003; accepted 1 January 2003
}

\begin{abstract}
Termination is an important and well-studied property for logic programs. However, almost all approaches for automated termination analysis focus on definite logic programs, whereas real-world Prolog programs typically use the cut operator. We introduce a novel pre-processing method which automatically transforms Prolog programs into logic programs without cuts, where termination of the cut-free program implies termination of the original program. Hence after this pre-processing, any technique for proving termination of definite logic programs can be applied. We implemented this pre-processing in our termination prover AProVE and evaluated it successfully with extensive experiments.
\end{abstract}

$K E Y W O R D S$ : automated termination analysis, cut, definite logic programs

\section{Introduction}

Automated termination analysis for logic programs has been widely studied, see, e.g., (Bruynooghe et al. 2007; Codish et al. 2005, De Schreye and Decorte 1994; Mesnard and Serebrenik 2007, Nguyen et al. 2010. Schneider-Kamp et al. 2009. Serebrenik and De Schreye 2005). Still, virtually all existing techniques only prove universal termination of definite logic programs, which do not use the cut "!". An exception is (Marchiori 1996), which transforms "safely typed" logic programs to term rewrite systems (TRSs). However, the resulting TRSs are complex and since there is no implementation of (Marchiori 1996), it is unclear whether they can be handled by existing TRS termination tools. Moreover, (Marchiori 1996)'s method

* Supported by the Deutsche Forschungsgemeinschaft (DFG) under grant GI 274/5-2, the DFG Research Training Group 1298 (AlgoSyn), and the Danish Natural Science Research Council. 
individual cuts to make their scope explicit. So a signature $\Sigma$ contains all predicate and function symbols and all labeled versions of the cut $\left\{!_{m} / 0 \mid m \in \mathbb{N}\right\}$. For simplicity we just consider terms $\mathcal{T}(\Sigma, \mathcal{V})$ and no atoms, i.e., we do not distinguish between predicate and function symbols. To ease the presentation, in the paper we exclude terms with cuts $!_{m}$ as proper subterms. A clause is a pair $H \leftarrow B$ where the head $H$ is from $\mathcal{T}(\Sigma, \mathcal{V})$ and the body $B$ is a sequence of terms from $\mathcal{T}(\Sigma, \mathcal{V})$. Let $\operatorname{Goal}(\Sigma, \mathcal{V})$ be the set of all such sequences, where $\square$ is the empty goal.

A program $\mathcal{P}$ (possibly with cut) is a finite sequence of clauses. Slice $(\mathcal{P}, t)$ are all clauses for $t$ 's predicate, i.e., $\operatorname{Slice}\left(\mathcal{P}, p\left(t_{1}, \ldots, t_{n}\right)\right)=\left\{c \mid c=" p\left(s_{1}, \ldots, s_{n}\right) \leftarrow B\right.$ " $\left.\in \mathcal{P}\right\}$.

A substitution $\sigma$ is a function $\mathcal{V} \rightarrow \mathcal{T}(\Sigma, \mathcal{V})$ and we often denote its application to a term $t$ by $t \sigma$ instead of $\sigma(t)$. As usual, $\operatorname{Dom}(\sigma)=\{X \mid X \sigma \neq X\}$ and Range $(\sigma)=\{X \sigma \mid X \in \operatorname{Dom}(\sigma)\}$. The restriction of $\sigma$ to $\mathcal{V}^{\prime} \subseteq \mathcal{V}$ is $\sigma \mid \mathcal{V}^{\prime}(X)=\sigma(X)$ if $X \in \mathcal{V}^{\prime}$, and $\left.\sigma\right|_{\mathcal{V}^{\prime}}(X)=X$ otherwise. A substitution $\sigma$ is the most general unifier (mgu) of $s$ and $t$ iff $s \sigma=t \sigma$ and, whenever $s \gamma=t \gamma$ for some $\gamma$, there exists a $\delta$ such that $X \gamma=X \sigma \delta$ for all $X \in \mathcal{V}(s) \cup \mathcal{V}(t)$. If $s$ and $t$ have no mgu, we write $s \not t$. Finally, to denote the term resulting from replacing all occurrences of a function symbol $f$ in a term $t$ by another function symbol $g$, we write $t[f / g]$.

Now we recapitulate the operational semantics of logic programming with cut. Compared to other formulations like (Andrews 2003, Billaud 1990, de Vink 1989: Kulas and Beierle 2000, Spoto 2000), the advantage of our formalization is that it is particularly suitable for an extension to classes of queries in Sect. 3 and 4. and for synthesizing cut-free programs in Sect. 5. A formal proof on the correspondence of our inference rules to the semantics of the Prolog ISO standard (Deransart et al. 1996) can be found in (Ströder 2010).

Our semantics is given by 7 inference rules. They operate on states which represent the current goal, and also the backtrack information that is needed to describe the effect of cuts. The backtrack information is given by a sequence of goals which are optionally labeled by the program clause that has to be applied to the goal next. Moreover, our states also contain explicit marks for the scope of a cut.

\section{Definition 1 (Concrete State)}

A concrete state is a sequence of elements from $\operatorname{Goal}(\Sigma, \mathcal{V}) \cup(\operatorname{Goal}(\Sigma, \mathcal{V}) \times \mathbb{N} \times \mathbb{N}) \cup$ $\left\{?_{n} \mid n \in \mathbb{N}\right\}$, where elements are separated by "|". State $(\Sigma, \mathcal{V})$ is the set of all states.

So an element of a state can be $Q \in \operatorname{Goal}(\Sigma, \mathcal{V})$; or a labeled goal $Q_{m}^{i} \in$ $\operatorname{Goal}(\Sigma, \mathcal{V}) \times \mathbb{N} \times \mathbb{N}$ representing that we must apply the $i$-th program clause to $Q$ next, where $m$ determines how a cut introduced by the $i$-th clause will be labeled; or $?_{m}$. Here, $?_{m}$ serves as a marker to denote the end of the scope of cuts $!_{m}$ labeled with $m$. Whenever a cut $!_{m}$ is reached, all elements preceding $?_{m}$ are discarded.

Now we express derivations in logic programming with cut by seven rules. Here, $S$ and $S^{\prime}$ are concrete states and the goal $Q$ may also be $\square$ (then " $t, Q$ " is $t$ ).

Definition 2 (Semantics with Concrete Inference Rules)

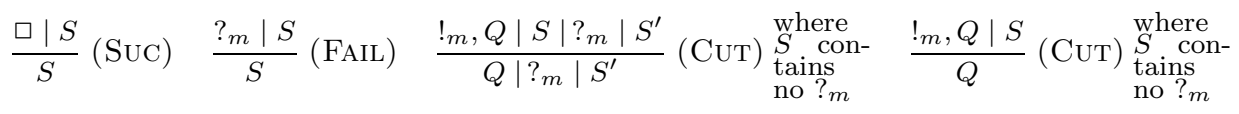




$$
\begin{aligned}
& \begin{array}{cl}
t, Q \mid S & \begin{array}{l}
\text { where } t \text { is neither a cut nor a variable, } m \\
\text { is greater than all previous marks, and } \\
\text { Slice }(\mathcal{P}, t)=\left\{c_{i_{1}}, \ldots, c_{i_{k}}\right\} \text { with } i_{1}< \\
\ldots<i_{k}
\end{array} \\
\hline(t, Q)_{m}^{i_{1}}|\ldots|(t, Q)_{m}^{i_{k}}\left|?_{m}\right| S & \text { CASE) }
\end{array} \\
& \frac{(t, Q)_{m}^{i} \mid S}{B_{i}^{\prime} \sigma, Q \sigma \mid S}(\text { Eval }) \begin{array}{l}
\begin{array}{l}
\text { where } \\
c_{i}=H_{i} \leftarrow B_{i}, \\
m g u\left(t, H_{i}\right)=\sigma \\
B_{i}^{\prime}=B_{i}\left[! / !_{m}\right] .
\end{array} \\
S
\end{array} \quad \begin{array}{l}
\text { where } \\
c_{i}=H_{i} \leftarrow B_{i} \\
\text { and } t \nsim H_{i} .
\end{array}
\end{aligned}
$$

The Suc rule is applicable if the first goal of our sequence could be proved. As we handle universal termination, we then have to backtrack to the next goal in the sequence. FAIL means that for the current $m$-th case analysis, there are no further backtracking possibilities. But the whole derivation does not have to fail, since the state $S$ may still contain further alternative goals which have to be examined.

To make the backtracking possibilities explicit, the resolution of a program clause with the first atom $t$ of the current goal is split into two operations. The CASE analysis determines which clauses could be applied to $t$ by slicing the program according to $t$ 's root symbol. It replaces the current goal $(t, Q)$ by a goal labeled with the index $i_{1}$ of the first such clause and adds copies of $(t, Q)$ labeled by the indices $i_{2}, \ldots, i_{k}$ of the other potentially applicable clauses as backtracking possibilities. Note that here, the top-down clause selection rule is taken into account. Additionally, these goals are labeled by a fresh mark $m \in \mathbb{N}$ that is greater than all previous marks, and $?_{m}$ is added at the end of the new backtracking goals to denote the scope of cuts. For instance, consider the program of Ex. 1 and the query $\operatorname{div}(0,0, Z)$. Here, we obtain the sequence depicted at the side. The CASE rule results in a state which represents a case analysis where we first try to apply the first

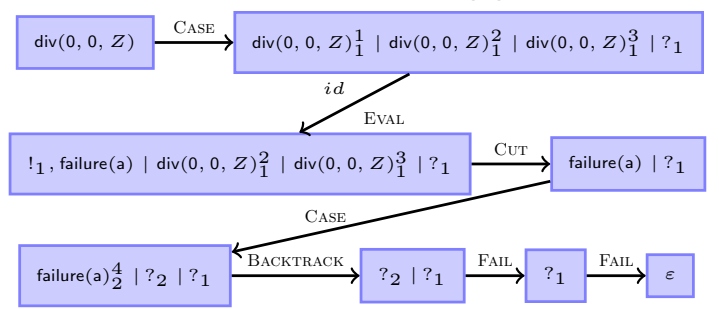
div-clause (1). When backtracking later on, we use clauses (2) and (3).

For a goal $(t, Q)_{m}^{i}$, if $t$ unifies with the head $H_{i}$ of the corresponding clause, we apply EvaL. This rule replaces $t$ by the body $B_{i}$ of the clause and applies the mgu $\sigma$ to the result. When depicting rule applications as trees, the corresponding edge is labeled with $\left.\sigma\right|_{\mathcal{V}(t)}$. All cuts occurring in $B_{i}$ are labeled with $m$. The reason is that if one reaches such a cut, then all further alternative goals up to $?_{m}$ are discarded.

If $t$ does not unify with $H_{i}$, we apply the BACKTRACK rule. Then, Clause $i$ cannot be used and we just backtrack to the next possibility in our backtracking sequence.

Finally, there are two CuT rules. The first rule removes all backtracking information on the level $m$ where the cut was introduced. Since the explicit scope is represented by $!_{m}$ and $?_{m}$, we have turned the cut into a local operation depending solely on the current state. Note that $?_{m}$ must not be deleted as the current goal $Q$ could still lead to another cut $!_{m}$. The second Cut rule is used if $?_{m}$ is missing (e.g., if a cut $!_{m}$ is already in the initial query). Later on, such states can also result from the additional PARALLEL inference rule which will be introduced in Sect. 4, We treat such states as if $?_{m}$ were added at the end of the backtracking sequence.

Note that these rules do not overlap, i.e., there is at most one rule that can be 
applied to any state. The only case where no rule is applicable is when the state is the empty sequence (denoted $\varepsilon$ ) or when the first goal starts with a variable.

The rules of Def. 2 define the semantics of logic programs with cut using states. They can also be used to define the semantics using derivations between goals: there is a derivation from the goal $Q$ to $Q^{\prime}$ in the program $\mathcal{P}\left(\right.$ denoted $\left.Q \vdash_{\mathcal{P}, \theta}^{*} Q^{\prime}\right)$ iff repeated application of our rules can transform the state ${ }^{1} Q$ to a state of the form $\bar{Q}^{\prime} \mid S$ for some $S$, and $Q^{\prime}$ results from $\bar{Q}^{\prime}$ by removing all labels. Moreover, $\theta=\theta_{1} \theta_{2} \ldots \theta_{n}$ where $\theta_{1}, \ldots, \theta_{n}$ are the mgu's used in those applications of the EvAL rule that led to $\bar{Q}^{\prime}$. We call $\left.\theta\right|_{\mathcal{V}(Q)}$ the corresponding answer substitution. If $\theta$ is not of interest, we write $\vdash_{\mathcal{P}}$ instead of $\vdash_{\mathcal{P}, \theta}$.

Consequently, our inference rules can be used for termination proofs: If there is an infinite derivation (w.r.t. $\vdash_{\mathcal{P}}$ ) starting in some goal $Q$, then there is also an infinite sequence of inference rule applications starting in the state $Q$, i.e., $Q$ is a "nonterminating state". Note that we distinguish derivations in logic programming (i.e., $Q \vdash_{\mathcal{P}} Q^{\prime}$ for goals $Q$ and $Q^{\prime}$ ) from sequences of states that result from application of the inference rules in Def. 2. If a state $S$ can be transformed into a state $S^{\prime}$ by such an inference rule, we speak of a "state-derivation".

\section{Abstract Derivations}

To represent classes of queries, we introduce abstract terms and a set $\mathcal{A}$ of abstract variables, where each $T \in \mathcal{A}$ represents a fixed but arbitrary term. $\mathcal{N}$ consists of all "ordinary" variables in logic programming. Then, as abstract terms we consider all terms from the set $\mathcal{T}(\Sigma, \mathcal{V})$ where $\mathcal{V}=\mathcal{N} \uplus \mathcal{A}$. Concrete terms are terms from $\mathcal{T}(\Sigma, \mathcal{N})$, i.e., terms containing no abstract variables. For any set $\mathcal{V}^{\prime} \subseteq \mathcal{V}$, let $\mathcal{V}^{\prime}(t)$ be the variables from $\mathcal{V}^{\prime}$ occurring in the term $t$.

To determine by which terms an abstract variable may be instantiated, we add a knowledge base $K B=(\mathcal{G}, \mathcal{U})$ to each state, where $\mathcal{G} \subseteq \mathcal{A}$ and $\mathcal{U} \subseteq \mathcal{T}(\Sigma, \mathcal{V}) \times$ $\mathcal{T}(\Sigma, \mathcal{V})$. The variables in $\mathcal{G}$ may only be instantiated by ground terms. And $\left(s, s^{\prime}\right) \in$ $\mathcal{U}$ means that we are restricted to instantiations $\gamma$ of the abstract variables where $s \gamma \not s^{\prime} \gamma$, i.e., $s$ and $s^{\prime}$ may not become unifiable when instantiating them with $\gamma$.

Definition 3 (Abstract State)

The set of abstract states $\operatorname{AState}(\Sigma, \mathcal{N}, \mathcal{A})$ is a set of pairs $(S ; K B)$ of a concrete state $S \in \operatorname{State}(\Sigma, \mathcal{N} \cup \mathcal{A})$ and a knowledge base $K B$.

A substitution $\gamma$ is a concretization of an abstract state if it respects the knowledge base $(\mathcal{G}, \mathcal{U})$. So first, $\gamma$ instantiates all abstract variables, i.e., $\operatorname{Dom}(\gamma)=\mathcal{A}$. Second, when applying $\gamma$, the resulting term must be concrete, i.e., $\mathcal{V}(\operatorname{Range}(\gamma)) \subseteq$ $\mathcal{N}$. Third, abstract variables from $\mathcal{G}$ may only be replaced by ground terms, i.e., $\mathcal{V}($ Range $(\gamma \mid \mathcal{G}))=\varnothing$. Fourth, for all pairs $\left(s, s^{\prime}\right) \in \mathcal{U}, s \gamma$ and $s^{\prime} \gamma$ must not unify.

Definition 4 (Concretization)

A substitution $\gamma$ is a concretization w.r.t. $(\mathcal{G}, \mathcal{U})$ iff $\operatorname{Dom}(\gamma)=\mathcal{A}, \mathcal{V}(\operatorname{Range}(\gamma)) \subseteq$ $\mathcal{N}, \mathcal{V}($ Range $(\gamma \mid \mathcal{G}))=\varnothing$, and $s \gamma \nsim s^{\prime} \gamma$ for all $\left(s, s^{\prime}\right) \in \mathcal{U}$. The set of concretizations of

\footnotetext{
${ }^{1}$ If $Q$ contains cuts, then the inference rules have to be applied to $Q\left[! / !_{1}\right]$ instead of $Q$.
} 
an abstract state $(S ; K B)$ is $\mathcal{C} o n(S ; K B)=\{S \gamma \mid \gamma$ is a concretization w.r.t. $K B\}$.

Example 2

Consider the abstract state which consists of the single goal sub $\left(T_{1}, T_{2}, T_{3}\right)$ and the knowledge base $\left(\left\{T_{1}, T_{2}\right\},\left\{\left(T_{1}, T_{3}\right)\right\}\right)$, with $T_{i} \in \mathcal{A}$ for all $i$. So here $\mathcal{G}=\left\{T_{1}, T_{2}\right\}$ and $\mathcal{U}$ only contains $\left(T_{1}, T_{3}\right)$. This represents all concrete states $\operatorname{sub}\left(t_{1}, t_{2}, t_{3}\right)$ where $t_{1}, t_{2}$ are ground terms and where $t_{1}$ and $t_{3}$ do not unify, i.e., $t_{3}$ does not match $t_{1}$. For example, $\operatorname{sub}(0,0, Z)$ is not represented as 0 and $Z$ unify. In contrast, $\operatorname{sub}(\mathrm{s}(0), \mathrm{s}(0), 0)$ and $\operatorname{sub}(0,0, s(0))$ are represented. Note that $\operatorname{sub}(\mathrm{s}(0), \mathrm{s}(0), 0)$ can be reduced to $\operatorname{sub}(0,0,0)$ using Clause (8) from Ex. 1. But Clause (8) cannot be applied to all concretizations. For example, the concrete state $\operatorname{sub}(0,0, s(0))$ is also represented by our abstract state, but here no clause is applicable.

Ex. 2 demonstrates that we need to adapt our inference rules to reflect that sometimes a clause can be applied only for some concretizations of the abstract variables, and to exploit the information from the knowledge base of the abstract state. We now adapt our inference rules to abstract states that represent sets of concrete states. The invariant of our rules is that all states represented by the parent node are terminating if all the states represented by its children are terminating.

Definition 5 (Sound Rules)

An abstract state is called terminating iff all its concretizations are terminating. A rule $\rho: \operatorname{AState}(\Sigma, \mathcal{N}, \mathcal{A}) \rightarrow 2^{\operatorname{AState}(\Sigma, \mathcal{N}, \mathcal{A})}$ is sound if $(S ; K B)$ is terminating whenever all $\left(S^{\prime} ; K B^{\prime}\right) \in \rho(S ; K B)$ are terminating.

The rules Suc, Fail, Cut, and CASE do not change the knowledge base and are, thus, straightforward to adapt. Here, $S \mid S^{\prime} ; K B$ stands for $\left(\left(S \mid S^{\prime}\right) ; K B\right)$.

Definition 6 (Abstract Inference Rules - Part 1 (Suc, FAIL, Cut, CASE))

$$
\begin{aligned}
& \frac{\square \mid S ; K B}{S ; K B}(\mathrm{SuC}) \quad \frac{?_{m} \mid S ; K B}{S ; K B}(\text { FAIL })
\end{aligned}
$$

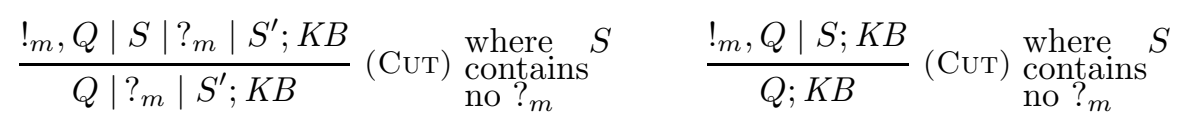

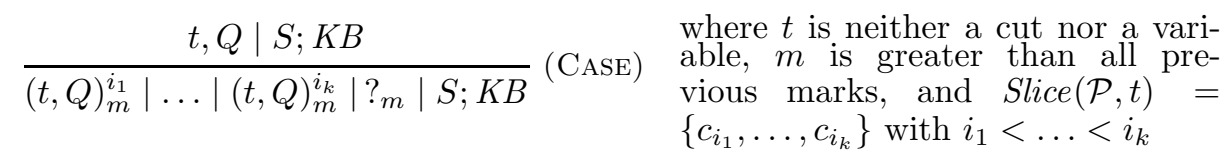

In Def. 2. we determined which of the rules Eval and BACKTRACK to apply by trying to unify the first atom $t$ with the head $H_{i}$ of the corresponding clause. But as demonstrated by Ex. 2, in the abstract case we might need to apply Eval for some concretizations and BACKTRACK for others. BACKTRACK can be used for all concretizations if $t$ does not unify with $H_{i}$ or if their mgu contradicts $\mathcal{U}$. This gives rise to the abstract BACKTRACK rule in the following definition. When the abstract BACKTRACK rule is not applicable, we still cannot be sure that $t \gamma$ unifies with $H_{i}$ for all concretizations $\gamma$. Thus, we have an abstract EvAL rule with two successor states that combines both the concrete EvAL and the concrete BACKTRACK rule. 
Definition 7 (Abstract Inference Rules - Part 2 (BACKTRACK, EvAL))

$$
\begin{gathered}
\frac{(t, Q)_{m}^{i} \mid S ; K B}{S ; K B} \text { (BACKTRACK) } \begin{array}{l}
\text { where } c_{i}=H_{i} \leftarrow B_{i} \text { and there is no con- } \\
\text { cretization } \gamma \text { w.r.t. } K B \text { such that } t \gamma \sim H_{i} .
\end{array} \\
\frac{(t, Q)_{m}^{i} \mid S ;(\mathcal{G}, \mathcal{U})}{B_{i}^{\prime} \sigma, Q \sigma|S \sigma|_{\mathcal{G}} ;\left(\mathcal{G}^{\prime},\left.\mathcal{U} \sigma\right|_{\mathcal{G}}\right) \quad S ;\left(\mathcal{G}, \mathcal{U} \cup\left\{\left(t, H_{i}\right)\right\}\right)}(\mathrm{EvAL})
\end{gathered}
$$

where $c_{i}=H_{i} \leftarrow B_{i}$ and $m g u\left(t, H_{i}\right)=\sigma$. W.l.o.g., $\mathcal{V}(\sigma(X))$ only contains fresh abstract variables for all $X \in \mathcal{V}$. Moreover, $\mathcal{G}^{\prime}=\mathcal{A}\left(\operatorname{Range}\left(\left.\sigma\right|_{\mathcal{G}}\right)\right)$ and $B_{i}^{\prime}=B_{i}\left[! / !_{m}\right]$.

In EvAL, w.l.o.g. we assume that $m g u\left(t, H_{i}\right)$ renames all variables to fresh abstract variables. This is needed to handle "sharing" effects correctly, i.e., to handle concretizations which introduce multiple occurrences of (concrete) variables, cf. (Schneider-Kamp et al. 2010). The knowledge base is updated differently for the successors corresponding to the concrete EVAL and BACKTRACK rule. For all concretizations corresponding to the second successor of EVAL, the concretization of $t$ does not unify with $H_{i}$. Hence, here we add the pair $\left(t, H_{i}\right)$ to the set $\mathcal{U}$.

Now consider concretizations $\gamma$ where $t \gamma$ and $H_{i}$ unify, i.e., concretizations $\gamma$ corresponding to the first successor of the EvaL rule. Then for any $T \in \mathcal{G}, T \gamma$ is a ground instance of $T \sigma$. Hence, we replace all $T \in \mathcal{G}$ by $T \sigma$, i.e., we apply $\left.\sigma\right|_{\mathcal{G}}$ to $\mathcal{U}$ and $S$. Now the new set $\mathcal{G}^{\prime}$ of abstract variables that may only be instantiated by ground terms is $\mathcal{A}\left(\right.$ Range $\left.\left(\left.\sigma\right|_{\mathcal{G}}\right)\right)$. As before, $t$ is replaced by the instantiated clause body $B_{i}$ where we label cuts with the number $m$ of the current CASE analysis.

Now any concrete derivation with the rules from Def. 2 can also be simulated with the abstract rules from Def. 6] and 7. But unfortunately, even for terminating goals, in general these rules yield an infinite tree. The reason is that there is no bound on the size of terms represented by the abstract variables and hence, the abstract EvAL rule can be applied infinitely often.

Example 3

Consider the 1-rule program

$$
\mathrm{p}(\mathrm{s}(X)) \leftarrow \mathrm{p}(X) .
$$

For queries of the form $\mathrm{p}(t)$ where $t$ is ground, the program terminates. However, the tree built using the abstract inference rules is obviously infinite.

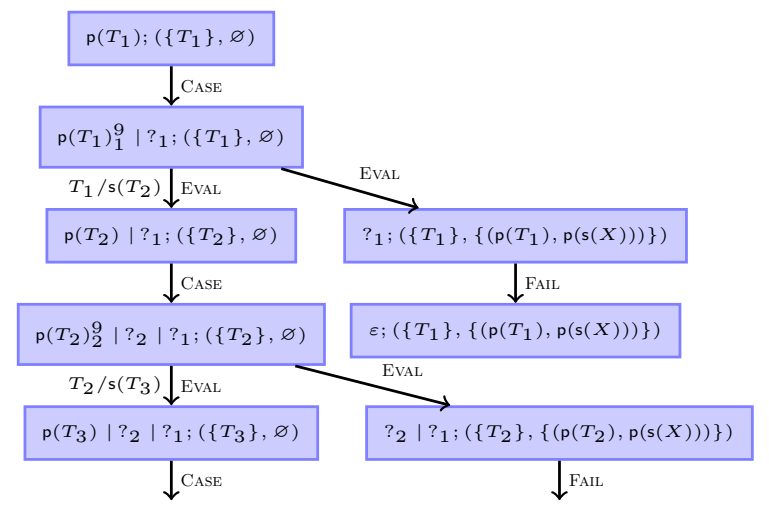

\section{From Trees to Graphs}

To obtain a finite graph instead of an infinite tree, we now introduce an additional INSTANCE rule which allows us to connect the current state $(S ; K B)$ with a previous state $\left(S^{\prime} ; K B^{\prime}\right)$, provided that the current state is an instance of the previous state. In other words, every concretization of $(S ; K B)$ must be a concretization of $\left(S^{\prime} ; K B^{\prime}\right)$. Still, INSTANCE is often not enough to obtain a finite graph. 
Example 4

We extend Ex. 3 by the following additional fact.

$$
\mathrm{p}(X) \text {. }
$$

For queries $\mathrm{p}(t)$ where $t$ is ground, the program still terminates. If we start with $\left(\mathrm{p}\left(T_{1}\right) ;\left(\left\{T_{1}\right\}, \varnothing\right)\right)$, then the CASE rule results in the state $\left(\mathrm{p}\left(T_{1}\right) \frac{\sqrt{9}}{1}\left|\mathrm{p}\left(T_{1}\right) \frac{\sqrt{10}}{1}\right| ?_{1}\right.$; $\left.\left(\left\{T_{1}\right\}, \varnothing\right)\right)$ and the Eval rule produces two new states, one of them being $\left(\mathrm{p}\left(T_{2}\right) \mid\right.$ $\mathrm{p}\left(\mathrm{s}\left(T_{2}\right) \frac{10}{10} \mid ?_{1} ;\left(\left\{T_{2}\right\}, \varnothing\right)\right)$.

To simplify states, from now on we will eliminate so-called non-active marks ${ }_{m}$ which occur as first or as last element in states. Eliminating $?_{m}$ from the beginning of a state is possible, as FAIL would also remove such a $?_{m}$. Eliminating $?_{m}$ from the end of a state is possible, as applying the first CUT rule to a state ending in $?_{m}$ is equivalent to applying the second CUT rule to the same state without $?_{m}$.

We will also reduce the knowledge base to just those abstract variables that occur in the state and remove pairs $\left(s, s^{\prime}\right)$ from $\mathcal{U}$ where $s \nsim s^{\prime}$. Still, $\left(\mathrm{p}\left(T_{2}\right)\right.$ $\mathrm{p}\left(\mathrm{s}\left(T_{2}\right) \frac{10}{10} ;\left(\left\{T_{2}\right\}, \varnothing\right)\right)$ is not an instance of the previous state $\left(\mathrm{p}\left(T_{1}\right) ;\left(\left\{T_{1}\right\}, \varnothing\right)\right)$ due to the added backtrack goal $\mathrm{p}\left(\mathrm{s}\left(T_{2}\right)\right) \frac{10}{1}$. Therefore, we now introduce a PARALLEL rule that allows us to split a backtracking sequence into separate problems. Now we obtain the graph on the right.

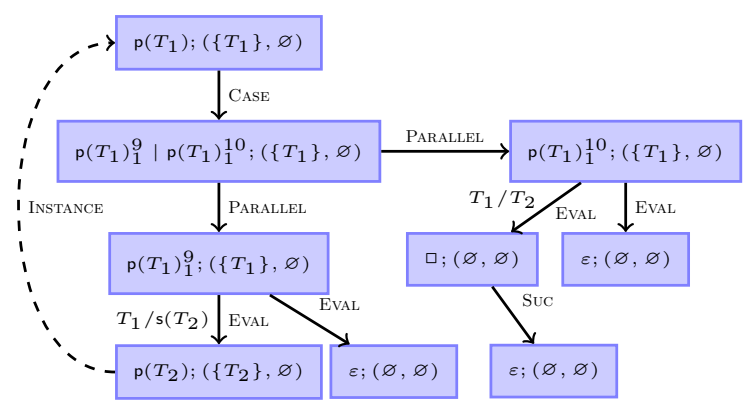

Clearly, PARALlEL may transform terminating into non-terminating states. But without further conditions, PARALLEL is not only "incomplete", but also unsound. Consider a state $\left(!_{2}\left|!_{1}\right| ?_{2} \mid \mathrm{p} ;(\varnothing, \varnothing)\right)$ for the program $\mathrm{p} \leftarrow \mathrm{p}$. The state is not terminating, as $!_{1}$ is not reachable. Thus, one eventually evaluates $p$. But if one splits the state into $\left.\left(!_{2} ; \varnothing, \varnothing\right)\right)$ and $\left(!_{1}\left|?_{2}\right| \mathrm{p} ;(\varnothing, \varnothing)\right)$, both new states terminate.

To solve this problem, in addition to the "active marks" (cf. Ex. 4) we introduce the notion of active cuts. The active cuts of a state $S$ are those $m \in \mathbb{N}$ where $!_{m}$ occurs in $S$ or where $!_{m}$ can be introduced by EvAL applied to a labeled goal $(t, q)_{m}^{i}$ occurring in $S$. Now the PARALLEL rule may only split a backtracking sequence into two parts $S$ and $S^{\prime}$ if the active cuts of $S$ and the active marks of $S^{\prime}$ are disjoint.

Definition 8 (Abstract Inference Rules - Part 3 (Instance, Parallel))

$\frac{S ;(\mathcal{G}, \mathcal{U})}{S^{\prime} ;\left(\mathcal{G}^{\prime}, \mathcal{U}^{\prime}\right)}($ Instance $) \quad \begin{aligned} & \text { if there is a } \mu \text { such that } S=S^{\prime} \mu,\left.\mu\right|_{\mathcal{N}} \text { is a variable } \\ & \text { renaming, } \mathcal{V}(T \mu) \subseteq \mathcal{G} \text { for all } T \in \mathcal{G}^{\prime}, \text { and } \mathcal{U}^{\prime} \mu \subseteq \mathcal{U}\end{aligned}$

$$
\frac{S \mid S^{\prime} ; K B}{S ; K B \quad S^{\prime} ; K B}(\text { PARAllel }) \quad \text { if } A C(S) \cap A M\left(S^{\prime}\right)=\varnothing
$$

The active cuts $A C(S)$ are all $m$ where $!_{m}$ is in $S$ or $(t, q)_{m}^{i}$ is in $S$ and $c_{i}$ 's body has a cut. The active marks $A M(S)$ are all $m$ where $S=S^{\prime}\left|?_{m}\right| S^{\prime \prime}$ and $S^{\prime} \neq \varepsilon, S^{\prime \prime} \neq \varepsilon$. 
Example 5

However, there are still examples where the graph cannot be "closed". Consider the program

$$
\mathrm{p}(\mathrm{s}(X)) \leftarrow \mathrm{p}(X), \mathrm{q} .
$$

q. (12)

For queries $\mathrm{p}(t)$ where $t$ is ground, the program again terminates. With Def. 6, 7, and 8, we obtain the infinite tree on the right. It never encounters an instance of a previous state, since each resolution with Clause (11) adds a q to the goal.

Thus, we introduce a final abstract SPLIT rule to split a state $(t, Q ; K B)$ into $(t ; K B)$ and a state $\left(Q \mu ; K B^{\prime}\right)$, where $\mu$ approximates the answer substitutions for $t$. The edge from $(t, Q ; K B)$ to $\left(Q \mu ; K B^{\prime}\right)$ is labeled with $\left.\mu\right|_{\mathcal{V}(t) \cup \mathcal{V}(Q)}$. To simplify the SpLIT rule, we only define it for backtracking sequences of one element. To obtain such a sequence, we can use the PARALLEL rule.

Definition 9 (Abstract Inference Rules - Part 4 (SPLIT))

$$
\begin{array}{cl}
t, Q ;(\mathcal{G}, \mathcal{U}) & \begin{array}{l}
\text { where } \mu \text { replaces all variables from } \mathcal{V} \backslash \mathcal{G} \\
\text { by fresh abstract variables and } \mathcal{G}^{\prime}=\mathcal{G} \cup \\
\text { Approx } G n d(t, \mu) .
\end{array}
\end{array}
$$

Here, ApproxGnd is defined as follows. We assume that we have a groundness analysis function Ground $d_{\mathcal{P}}: \Sigma \times 2^{\mathbb{N}} \rightarrow 2^{\mathbb{N}}$, see, e.g., (Howe and King 2003). If $p$ is an $n$-ary predicate, $\left\{i_{1}, \ldots, i_{m}\right\} \subseteq\{1, \ldots, n\}$, and $\operatorname{Ground}_{\mathcal{P}}\left(p,\left\{i_{1}, \ldots, i_{m}\right\}\right)=$ $\left\{j_{1}, \ldots, j_{k}\right\}$, then any successful derivation $p\left(t_{1}, \ldots, t_{n}\right) \vdash_{\mathcal{P}, \theta}^{*} \square$ where $t_{i_{1}}, \ldots, t_{i_{m}}$ are ground will lead to an answer substitution $\theta$ such that $t_{j_{1}} \theta, \ldots, t_{j_{k}} \theta$ are ground. So Ground $_{\mathcal{P}}$ approximates which positions of $p$ will become ground if the "input" positions $i_{1}, \ldots, i_{m}$ are ground. Now if $t=p\left(t_{1}, \ldots, t_{n}\right)$ is an abstract term where $t_{i_{1}}, \ldots, t_{i_{m}}$ are ground in every concretization (i.e., all their variables are from $\mathcal{G}$ ), then Approx $G n d(t, \mu)$ returns the $\mu$-renamings of all abstract variables that will be ground in every successful derivation starting from a concretization of $t$. Thus, Approx $\operatorname{Gnd}(t, \mu)$ contains the abstract variables of $t_{j_{1}} \mu, \ldots, t_{j_{k}} \mu$. So formally

$$
\operatorname{Approx} G n d\left(p\left(t_{1}, \ldots, t_{n}\right), \mu\right)=\left\{\mathcal{A}\left(t_{j} \mu\right) \mid j \in \operatorname{Ground}_{\mathcal{P}}\left(p,\left\{i \mid \mathcal{V}\left(t_{i}\right) \subseteq \mathcal{G}\right\}\right)\right\}
$$

\section{Example 6}

To illustrate Def. 9, regard the program of Ex. 11 and the state $\left(\operatorname{sub}\left(T_{5}, T_{6}, T_{8}\right)\right.$, $\left.\operatorname{div}\left(T_{8}, T_{6}, T_{7}\right) ;\left(\left\{T_{5}, T_{6}\right\}, \mathcal{U}\right)\right)$ with $T_{5}, T_{6}, T_{7}, T_{8} \in \mathcal{A}$. (This state will occur in the termination proof of div, cf. Ex. 7) We have $\mathcal{G}=\left\{T_{5}, T_{6}\right\}$ and hence if $\operatorname{sub}\left(t_{1}, t_{2}, t_{3}\right)$ is $\operatorname{sub}\left(T_{5}, T_{6}, T_{8}\right)$, then $\operatorname{Ground}_{\mathcal{P}}\left(\operatorname{sub},\left\{i \mid \mathcal{V}\left(t_{i}\right) \subseteq \mathcal{G}\right\}\right)=\operatorname{Ground}_{\mathcal{P}}(\operatorname{sub},\{1,2\})=$ $\{1,2,3\}$. In other words, if the first two arguments of sub are ground and the derivation is successful, then the answer substitution also instantiates the third argument to a ground term. Since $\mu$ only renames variables outside of $\mathcal{G}$, we have $\mu=$ $\left\{T_{7} / T_{9}, T_{8} / T_{10}\right\}$. So Approx $\operatorname{And}\left(\operatorname{sub}\left(T_{5}, T_{6}, T_{8}\right), \mu\right)=\left\{\mathcal{A}\left(t_{1} \mu\right), \mathcal{A}\left(t_{2} \mu\right), \mathcal{A}\left(t_{3} \mu\right)\right\}=$ $\left\{T_{5} \mu, T_{6} \mu, T_{8} \mu\right\}=\left\{T_{5}, T_{6}, T_{10}\right\}$. So the SpLIT rule transforms the current state 
to $\left(\operatorname{sub}\left(T_{5}, T_{6}, T_{8}\right) ;\left(\left\{T_{5}, T_{6}\right\}, \mathcal{U}\right)\right)$ and $\left(\operatorname{div}\left(T_{10}, T_{6}, T_{9}\right) ;\left(\left\{T_{5}, T_{6}, T_{10}\right\}, \mathcal{U} \mu\right)\right)$ where one can eliminate $T_{5}$ from the new groundness set $\mathcal{G}^{\prime}$.

With the additional SpLiT rule, we can always obtain finite graphs instead of infinite trees. (This will be proved in Thm. 2) Thus, no further rules are needed. As depicted on the right, now we can also close the graph for Ex. 5's program.

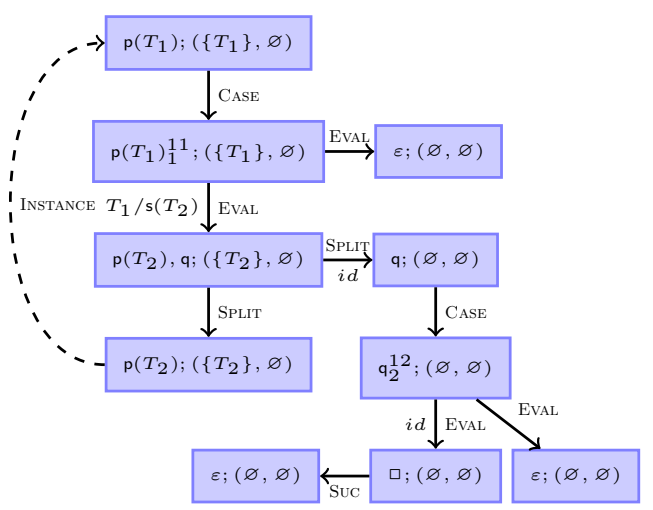

Thm. 1 proves the soundness of all our abstract inference rules. In other words, if all children of a node are terminating, then the node is terminating as well.

Theorem 1 (Soundness of the Abstract Inference Rules) The inference rules from Def. 6, 7, 8, and 9 are sound. ${ }^{2}$

\section{From Termination Graphs to Logic Programs}

Now we introduce termination graphs as a subclass of the graphs obtained by Def. 6 . 7. 8, 9. Then we show how to extract cut-free programs from termination graphs.

\section{Definition 10 (Termination Graph)}

A finite graph built from an initial state $(S ; K B)$ using Def. 6, 7, 8, and 9 is a termination graph iff there is no cycle consisting only of InSTANCE edges and all leaves are of the form $\left(\varepsilon ; K B^{\prime}\right)$ or $\left(X, Q \mid S ; K B^{\prime}\right)$ with $X \in \mathcal{V}$. If there are no leaves of the form $\left(X, Q \mid S ; K B^{\prime}\right)$, then the graph is "proper".

We want to generate clauses for the loops in the termination graph and show their termination. Thus, there should be no cycles consisting only of INSTANCE edges, as they would lead to trivially non-terminating clauses. Moreover, the only leaves may be nodes where no inference rule is applicable anymore (i.e., the graph must be "fully expanded"). For example, the graph at the end of Sect. 4 is a termination graph. Thm. 2 shows that termination graphs can always be obtained automatically.

Theorem 2 (Existence of Termination Graphs)

For any program $\mathcal{P}$ and abstract state $(S ; K B)$, there exists a termination graph.

\section{Example 7}

For the program from Ex. 1 we obtain the termination graph below. Here, $\mathcal{U}=$ $\left\{\left(\operatorname{div}\left(T_{5}, T_{6}, T_{3}\right), \operatorname{div}(X, 0, Z)\right),\left(\operatorname{div}\left(T_{5}, T_{6}, T_{3}\right), \operatorname{div}(0, Y, Z)\right)\right\}$ results from exploiting the cuts. $\mathcal{U}$ implies that neither $T_{6}$ nor $T_{5}$ unify with 0 . Thus, only Clause (8) is applicable to evaluate the state in Node D. This is crucial for termination, because in D, sub's result $T_{8}$ is always smaller than sub's input argument $T_{5}$ and therefore, div's first argument in Node C is smaller than div's first argument in Node A.

Remember that our goal is to show termination of the graph's initial state. Since

\footnotetext{
${ }^{2}$ For all proofs, we refer to (Schneider-Kamp et al. 2010).
} 
the graph only has leaves $(\varepsilon ; K B)$ that are clearly terminating, by soundness of the inference rules, it remains to prove that there is no state-derivation corresponding to an infinite traversal of the cycles in the graph. So in our example, we have to show that the INSTANCE edges for div and sub cannot be traversed infinitely often.

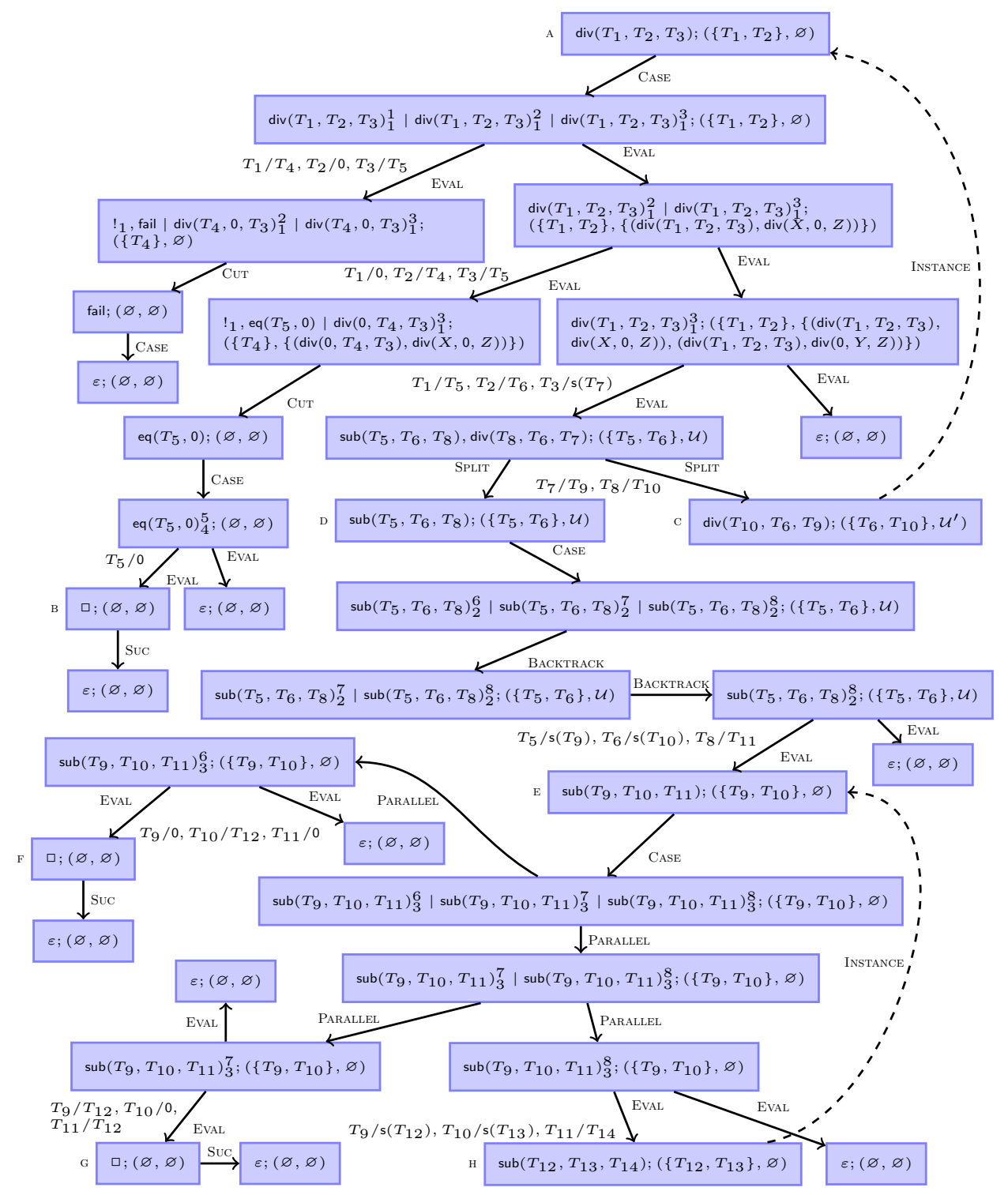

We now synthesize a cut-free program from the termination graph. This program has the following property: if there is a state-derivation from a concretization of one state to a concretization of another state which may be crucial for non-termination, then there is a corresponding derivation in the obtained cut-free program.

More precisely, we build clauses for all clause paths. For a termination graph $G$, let $\operatorname{Instance}(G)$ denote all nodes of $G$ to which the rule Instance has been 
applied (i.e., C and $\mathrm{H}$ in our example). The sets $\operatorname{Split}(G)$ and $\operatorname{SuC}(G)$ are defined analogously. For any node $n$, let $\operatorname{Succ}(i, n)$ denote the $i$-th child of $n$. Clause paths are paths in the graph that start in the root node, in the successor node of an INSTANCE node, or in the left child of a SPLIT node and that end in a SuC or INSTANCE node or in the left child of an INSTANCE or SPLIT node.

Definition 11 (Clause Path)

A path $\pi=n_{1} \ldots n_{k}$ in $G$ is a clause path iff $k>1$ and

- $n_{1} \in \operatorname{Succ}(1, \operatorname{Instance}(G) \cup \operatorname{Split}(G))$ or $n_{1}$ is the root of $G$,

- $n_{k} \in \operatorname{SuC}(G) \cup \operatorname{Instance}(G) \cup \operatorname{Succ}(1, \operatorname{Instance}(G) \cup \operatorname{Split}(G))$,

- for all $1 \leq j<k$, we have $n_{j} \notin \operatorname{Instance}(G)$, and

- for all $1<j<k$, we have $n_{j} \notin \operatorname{Succ}(1, \operatorname{Instance}(G) \cup \operatorname{Split}(G))$.

Since we only want finitely many clause paths, they may not traverse InSTANCE edges. Clause paths may also not follow left successors of INSTANCE or SPLIT. Instead, we create new clause paths starting at these nodes. In our example, we have clause paths from A to B, A to C, A to D, D to E, E to F, E to G, and E to H.

To obtain a cut-free logic program, we construct one clause for each clause path $\pi=n_{1} \ldots n_{k}$. The head of the new clause corresponds to $n_{1}$ where we apply the relevant substitutions between $n_{1}$ and $n_{k}$. The last body atom corresponds to $n_{k}$. The intermediate body atoms correspond to those nodes that are left children of those $n_{i}$ which are from $\operatorname{SpLiT}(G)$. Note that we apply the relevant substitutions between $n_{i}$ and $n_{k}$ to the respective intermediate body atom as well.

In our example, the path from A to B is labeled by the substitution $\sigma=\left\{T_{1} / 0\right.$, $\left.T_{2} / T_{4}, T_{3} / 0, T_{5} / 0\right\}$. Hence, we obtain the fact $\operatorname{div}_{\mathrm{A}}\left(T_{1}, T_{2}, T_{3}\right) \sigma=\operatorname{div}_{\mathrm{A}}\left(0, T_{4}, 0\right)$. We always use a new predicate symbol when translating a node into an atom of a new clause (i.e., $\operatorname{div}_{\mathrm{A}}$ is fresh). InSTANCE nodes are the only exception. There, we use the same predicate symbol both for the INSTANCE node and its successor.

For the path from A to C, we have the substitution $\sigma^{\prime}=\left\{T_{1} / T_{5}, T_{2} / T_{6}, T_{3} / \mathrm{s}\left(T_{9}\right)\right.$, $\left.T_{7} / T_{9}, T_{8} / T_{10}\right\}$. Right children of SpLIT nodes can only be reached if the goal in the left SPLIT-child was successful. So $\operatorname{sub}\left(T_{5}, T_{6}, T_{8}\right) \sigma^{\prime}$ must be derived to $\square$ before the derivation can continue with div. Thus, we obtain the new clause $\operatorname{div}_{\mathrm{A}}\left(T_{5}, T_{6}, \mathrm{~s}\left(T_{9}\right)\right) \leftarrow \operatorname{sub}_{\mathrm{D}}\left(T_{5}, T_{6}, T_{10}\right), \operatorname{div}_{\mathrm{A}}\left(T_{10}, T_{6}, T_{9}\right)$. Note that we used the same symbol $\operatorname{div}_{\mathrm{A}}$ for both occurrences of div as they are linked by an INSTANCE edge.

Continuing in this way, we obtain the following logic program for which we have to show termination w.r.t. the set of queries $\left\{\operatorname{div}_{\mathrm{A}}\left(t_{1}, t_{2}, t_{3}\right) \mid t_{1}, t_{2}\right.$ are ground $\}$, as specified by the knowledge base in the root node $\mathrm{A}$.

$$
\begin{aligned}
\operatorname{div}_{\mathrm{A}}\left(0, T_{4}, 0\right) . & \\
\operatorname{div}_{\mathrm{A}}\left(T_{5}, T_{6}, \mathrm{~s}\left(T_{9}\right)\right) & \leftarrow \operatorname{sub}_{\mathrm{D}}\left(T_{5}, T_{6}, T_{10}\right), \operatorname{div}_{\mathrm{A}}\left(T_{10}, T_{6}, T_{9}\right) . \\
\operatorname{div}_{\mathrm{A}}\left(T_{5}, T_{6}, \mathrm{~s}\left(T_{7}\right)\right) & \leftarrow \operatorname{sub}_{\mathrm{D}}\left(T_{5}, T_{6}, T_{8}\right) . \\
\operatorname{sub}_{\mathrm{D}}\left(\mathrm{s}\left(T_{9}\right), \mathrm{s}\left(T_{10}\right), T_{11}\right) & \leftarrow \operatorname{sub}_{\mathrm{E}}\left(T_{9}, T_{10}, T_{11}\right) . \\
\operatorname{sub}_{\mathrm{E}}\left(0, T_{12}, 0\right) . & \\
\operatorname{sub}_{\mathrm{E}}\left(T_{12}, 0, T_{12}\right) . & \\
\operatorname{sub}_{\mathrm{E}}\left(\mathrm{s}\left(T_{12}\right), \mathrm{s}\left(T_{13}\right), T_{14}\right) & \leftarrow \operatorname{sub}_{\mathrm{E}}\left(T_{12}, T_{13}, T_{14}\right) .
\end{aligned}
$$

Virtually all existing methods and tools for proving termination of logic programs succeed on this definite logic program. Hence, by our pre-processing technique, 
termination of programs with cut like Ex. 1 can be proved automatically.

In general, to convert a node $n$ into an atom, we use a function $\operatorname{Ren}$. $\operatorname{Ren}(n)$ has the form $p_{n}\left(X_{1}, \ldots, X_{n}\right)$ where $p_{n}$ is a fresh predicate symbol for the node $n$ (except if $n$ is an Instance node) and $X_{1}, \ldots, X_{n}$ are all variables in $n$. This renaming allows us to use different predicate symbols for different nodes. For example, the cut-free logic program above would not terminate if we identified $\operatorname{sub}_{\mathrm{D}}$ and $\mathrm{sub}_{\mathrm{E}}$. The reason is that sub $b_{D}$ only succeeds if its first and second argument start with "s". Hence, if the intermediate body atom $\operatorname{sub}_{\mathrm{D}}\left(T_{5}, T_{6}, T_{10}\right)$ of Clause (13) succeeds, then the "number $T_{10}$ " will always be strictly smaller than the "number $T_{5}$ ". Finally, Ren allows us to represent a whole state by just one atom, even if this state consists of a non-atomic goal or a backtracking sequence with several elements.

The only remaining problem is that paths may contain evaluations for several alternative backtracking goals of the same case analysis. Substitutions that correspond to "earlier" alternatives must not be regarded when instantiating the head of the new clause. The reason is that backtracking undoes the substitutions of previous evaluations. Thus, we collect the substitutions on the path starting with the substitution applied last. Here, we always keep track of the mark $d$ corresponding to the last EvAL node. Substitutions that belong to earlier alternatives of the current case analysis are disregarded when constructing the new cut-free program. These earlier alternatives can be identified easily, since they have marks $m$ with $m \geq d$.

\section{Example 5}

Consider the following program and the termination graph for the state $\left(\mathrm{p}\left(T_{1}\right) ;(\varnothing, \varnothing)\right)$ on the side. Here, we omitted the knowledge bases to ease readability.

$$
\begin{array}{lll}
\mathrm{p}(\mathrm{f}(X)) \leftarrow \mathrm{q}(X) . & (14) & \mathrm{q}(\mathrm{a}) .(16) \\
\mathrm{p}(\mathrm{g}(X)) \leftarrow \mathrm{r}(X) . & (15) & \mathrm{r}(\mathrm{b}) . \quad(17)
\end{array}
$$

This graph contains clause paths from A to B and from A to C. For every clause path, we collect the relevant substitutions step by step, starting from the end of the path. So for the first clause path we start with $\left\{T_{2} / a\right\}$. This substitution results from an EvAL node for the goal $\mathrm{q}\left(T_{2}\right) \frac{16}{2}$ with mark $d=2$. Hence, for the first clause path we only collect further substitutions that result from EvAL nodes with marks smaller than $d=2$. Since the next substitution $\left\{T_{1} / \mathrm{f}\left(T_{2}\right)\right\}$ results from an EVAL node with mark 1 , we finally obtain $\left\{T_{1} / \mathrm{f}\left(T_{2}\right)\right\} \circ\left\{T_{2} / \mathrm{a}\right\}$ which leads to the fact $p(f(a))$ in the resulting logic program. For the second clause path from A to $\mathrm{C}$, we start with $\left\{T_{3} / \mathrm{b}\right\}$ which results from an EvAL node with mark $d=3$. When moving upwards in the tree, the substitution $\left\{T_{1} / \mathrm{g}\left(T_{3}\right)\right\}$ also has to be collected, since it results from an EvAL node with

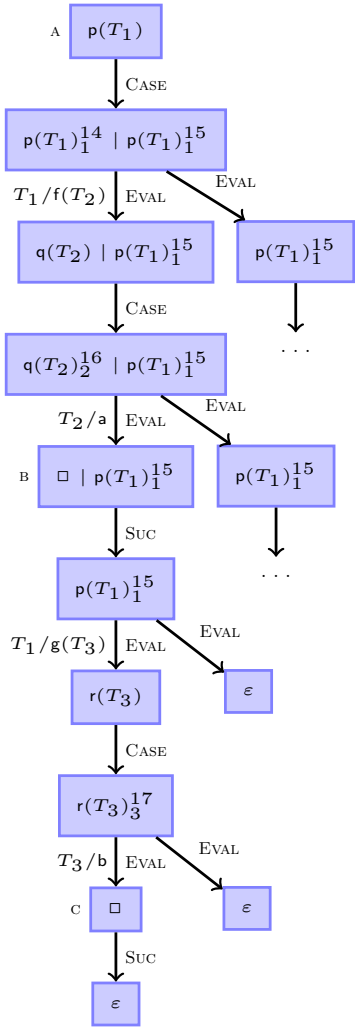
mark 1. Thus, we now set $d=1$. When moving upwards, we reach further substitutions, but they result from EvAL nodes with marks 2 and 1. These substitutions 
are not collected, since they correspond to earlier alternatives of this case analysis. Hence, we just obtain the substitution $\left\{T_{1} / \mathrm{g}\left(T_{3}\right)\right\} \circ\left\{T_{3} / \mathrm{b}\right\}$ for the second clause path, which yields the fact $\mathrm{p}(\mathrm{g}(\mathrm{b}))$ in the resulting logic program.

If we disregarded the marks when collecting substitutions, the second clause path would result in $\left\{T_{1} / \mathfrak{f}\left(T_{2}\right)\right\} \circ\left\{T_{2} / \mathrm{a}\right\} \circ\left\{T_{1} / \mathrm{g}\left(T_{3}\right)\right\} \circ\left\{T_{3} / \mathrm{b}\right\}$ instead. But then we would get the same fact $\mathrm{p}(\mathrm{f}(\mathrm{a}))$ as from the first clause path. So the new logic program would not simulate all derivations represented in the termination graph.

Now we formally define the cut-free logic program $\mathcal{P}_{G}$ and the corresponding class of queries $\mathcal{Q}_{G}$ resulting from a termination graph $G$. If $\mathcal{P}_{G}$ is terminating for all queries from $\mathcal{Q}_{G}$, then the root state of $G$ is terminating w.r.t. the original logic program (possibly containing cuts).

Definition 12 (Logic Programs and Queries from Termination Graph)

Let $G$ be a termination graph whose root $n$ is $\left(p\left(T_{1}, \ldots, T_{m}\right),\left(\left\{T_{i_{1}}, \ldots, T_{i_{k}}\right\}, \varnothing\right)\right)$. We define $\mathcal{P}_{G}=\bigcup_{\pi \text { clause path in } G}$ Clause $(\pi)$ and $\mathcal{Q}_{G}=\left\{p_{n}\left(t_{1}, \ldots, t_{m}\right) \mid t_{i_{1}}, \ldots, t_{i_{k}}\right.$ are ground $\}$. Here, $p_{n}$ is a new predicate which results from translating the node $n$ into a clause. For a path $\pi=n_{1} \ldots n_{k}$, let $\operatorname{Clause}(\pi)=\operatorname{Ren}\left(n_{1}\right) \sigma_{\pi, \infty} \leftarrow I_{\pi}, \operatorname{Ren}\left(n_{k}\right)$. For $n \in \operatorname{Suc}(G), \operatorname{Ren}(n)$ is $\square$ and for $n \in \operatorname{Instance}(G)$, it is $\operatorname{Ren}(\operatorname{Succ}(1, n)) \mu$ where $\mu$ is the substitution associated with the Instance node $n$. Otherwise, $\operatorname{Ren}(n)$ is $p_{n}(\mathcal{V}(n))$ where $p_{n}$ is a fresh predicate symbol and $\mathcal{V}(S ; K B)=\mathcal{V}(S)$.

Finally, $\sigma_{\pi, d}$ with $d \in \mathbb{N} \cup\{\infty\}$ and $I_{\pi}$ are defined as follows. Here for a path $\pi=n_{1} \ldots n_{j}$, the substitutions $\mu$ and $\sigma$ are the labels on the outgoing edge of $n_{j-1} \in \operatorname{SpLit}(G)$ and $n_{j-1} \in \operatorname{EvaL}(G)$, respectively, and the mark $m$ results from the corresponding node $n_{j-1}=\left((t, Q)_{m}^{i} \mid S ; K B\right)$.

$$
\begin{gathered}
\sigma_{n_{1} \ldots n_{j}, d}= \begin{cases}i d & \text { if } j=1 \\
\sigma_{n_{1} \ldots n_{j-1}, d} \mu & \text { if } n_{j-1} \in \operatorname{SputT}(G), n_{j}=\operatorname{Succ}\left(2, n_{j-1}\right) \\
\sigma_{n_{1} \ldots n_{j-1}, m} \sigma & \text { if } n_{j-1} \in \operatorname{EvaL}(G), n_{j}=\operatorname{Succ}\left(1, n_{j-1}\right), \text { and } d>m \\
\left.\sigma_{n_{1} \ldots n_{j-1}, d} \sigma\right|_{\mathcal{G}} & \text { if } n_{j-1} \in \operatorname{EvaL}(G), n_{j}=\operatorname{Succ}\left(1, n_{j-1}\right), \text { and } d \leq m \\
\sigma_{n_{1} \ldots n_{j-1}, d} & \text { otherwise }\end{cases} \\
I_{n_{j} \ldots n_{k}}= \begin{cases}\square & \text { if } j=k \\
\operatorname{Ren}\left(\operatorname{Succ}\left(1, n_{j}\right)\right) \sigma_{n_{j} \ldots n_{k}, \infty}, I_{n_{j+1} \ldots n_{k}} & \text { if } n_{j} \in \operatorname{SpLiT}(G), n_{j+1}=\operatorname{Succ}\left(2, n_{j}\right) \\
I_{n_{j+1} \ldots n_{k}} & \text { otherwise }\end{cases}
\end{gathered}
$$

So if $n_{j-1}$ is a SPLIT node, then one has to "collect" the corresponding substitution $\mu$ when constructing the overall substitution $\sigma_{n_{1} \ldots n_{j}, d}$ for the path. If $n_{j-1}$ is an Eval node for the $m$-th case analysis and $n_{j}$ is its left successor, then the construction of $\sigma_{n_{1} \ldots n_{j}, d}$ depends on whether we have already collected a corresponding substitution for the current case analysis $m$. If $m$ is smaller than the mark $d$ for the last case analysis which contributed to the substitution, then the corresponding substitution $\sigma$ of the Eval rule is collected and $d$ is set to $m$. Otherwise (if $d \leq m$ ), one only collects the part $\left.\sigma\right|_{\mathcal{G}}$ of the substitution that concerns those abstract variables that stand for ground terms. The definition of the intermediate body atoms $I_{\pi}$ ensures that derivations in $\mathcal{P}_{G}$ only reach the second child of a SPLIT node if the first child of the SPLIT node could successfully be proved.

Thm. 3 proves the soundness of our approach. So termination of the cut-free program $\mathcal{P}_{G}$ implies termination of the original program $\mathcal{P}$. (However as shown in 
(Schneider-Kamp et al. 2010), the converse does not hold.)

Theorem 3 (Soundness)

Let $G$ be a proper termination graph for $\mathcal{P}$ whose root is $\left(p\left(T_{1}, \ldots, T_{m}\right),\left(\left\{T_{i_{1}}, \ldots, T_{i_{k}}\right\}\right.\right.$, $\varnothing)$ ). If $\mathcal{P}_{G}$ terminates for all queries in $\mathcal{Q}_{G}$, then all concretizations of $G$ 's root state have only finite state-derivations. In other words, then all queries from the set $\left\{p\left(t_{1}, \ldots, t_{m}\right) \mid t_{i_{1}}, \ldots, t_{i_{k}}\right.$ are ground $\}$ terminate w.r.t. $\mathcal{P}$.

\section{Experiments and Conclusions}

We introduced a pre-processing method to eliminate cuts. Afterwards, any technique for proving universal termination of logic programming can be applied. Thus, termination of logic programs with cuts can now be analyzed automatically.

We implemented this pre-processing in our tool AProVE (Giesl et al. 2006) and performed extensive experiments which show that now we can indeed prove termination of typical logic programs with cut fully automatically. The implementation is not only successful for programs like Ex. 1, but also for programs using operators like negation as failure or if then else which can be expressed using cuts. While AProVE was already very powerful for termination analysis of definite logic programs (Schneider-Kamp et al. 2009), our pre-processing method strictly increases its power. For our experiments, we used the Termination Problem Database (TPDB) of the annual International Termination Competition. ${ }^{3}$ Since up to now, no tool had special support for cuts, the previous versions of the TPDB did not contain any programs with cuts. Therefore, we took existing cut-free examples from the TPDB and added cuts in a natural way. In this way, we extended the TPDB by 104 typical programs with cuts (directory LP/CUT). Of these, 10 are known to be non-terminating. Up to now, termination tools treated cuts by simply ignoring them and by trying to prove termination of the program that results from removing the cuts. This is sensible, since cuts are not always needed for termination. Indeed, a version of AProVE that ignores cuts and does not use our pre-processing can show termination of 10 of the 94 potentially terminating examples. Other existing termination tools would not yield much better results, since AProVE is already the most powerful tool for definite logic programming (as shown by the experiments in (Schneider-Kamp et al. 2009)) and since most of the remaining 84 examples do not terminate anymore if one removes the cut. In contrast, with our new pre-processing, AProVE proves termination of 78 examples (i.e., $83 \%$ of the potentially terminating examples). This shows that our contributions are crucial for termination analysis of logic programs with cuts. Nevertheless, there is of course room for further improvements (e.g., one could develop alternative techniques to generate cut-free clauses from the termination graph in order to improve the performance on examples which encode existential termination). To experiment with our implementation and for further details, we refer to http://aprove.informatik.rwth-aachen.de/eval/Cut/.

Acknowledgements. We thank the referees for many helpful remarks.

\footnotetext{
3 http://termination-portal.org/wiki/Termination_Competition
} 


\section{References}

Andrews, J. H. 2003. The witness properties and the semantics of the Prolog cut. TPLP 3, 1, 1-59.

Apt, K. R. 1997. From Logic Programming to Prolog. Prentice Hall, London.

Billaud, M. 1990. Simple operational and denotational semantics for Prolog with cut. Theor. Comp. Sc. 71, 2, 193-208.

Bruynooghe, M., Codish, M., Gallagher, J. P., Genaim, S., and Vanhoof, W. 2007. Termination analysis of logic programs through combination of type-based norms. ACM TOPLAS 29, 2.

Codish, M., Lagoon, V., and Stuckey, P. J. 2005. Testing for termination with monotonicity constraints. In ICLP '05. LNCS 3668. 326-340.

Cousot, P. And Cousot, R. 1992. Abstract interpretation and application to logic programs. J. Log. Prog. 13, 2-3, 103-179.

De Schreye, D. And Decorte, S. 1994. Termination of logic programs: The neverending story. J. Log. Prog. 19,20, 199-260.

DE Vink, E. P. 1989. Comparative semantics for Prolog with cut. Sci. Comp. Prog. 13, 1, $237-264$.

Deransart, P., Ed-Dbali, A., And Cervoni, L. 1996. Prolog: The Standard. Springer, New York.

Filé, G. AND Rossi, S. 1993. Static analysis of Prolog with cut. In LPAR '93. LNAI 698. $134-145$.

Giesl, J., Schneider-Kamp, P., And Thiemann, R. 2006. AProVe 1.2: Automatic termination proofs in the dependency pair framework. In IJCAR '06. LNAI 4130. 281286.

Giesl, J., Swiderski, S., Schneider-Kamp, P., and Thiemann, R. 2006. Automated termination analysis for Haskell: From term rewriting to programming languages. In RTA '06. LNCS 4098. 297-312.

Howe, J. M. And KIng, A. 2003. Efficient groundness analysis in Prolog. TPLP 3, 1, 95-124.

Kulas, M. And Beierle, C. 2000. Defining standard Prolog in rewriting logic. In WRLA '00. ENTCS 36.

Le Charlier, B., Rossi, S., And Van Hentenryck, P. 1994. An abstract interpretation framework which accurately handles Prolog search-rule and the cut. In ILPS '94. MIT Press, 157-171.

Marchiori, M. 1996. Proving existential termination of normal logic programs. In AMAST' '96. LNCS 1101. 375-390.

Mesnard, F. And Serebrenik, A. 2007. Recurrence with affine level mappings is P-time decidable for $\operatorname{CLP}(\mathrm{R})$. TPLP 8, 1, 111-119.

Mogensen, T. Æ. 1996. A semantics-based determinacy analysis for Prolog with cut. In Ershov Memorial Conference. LNCS 1181. 374-385.

Nguyen, M. T., De Schreye, D., Giesl, J., And Schneider-Kamp, P. 2010. Polytool: Polynomial interpretations as a basis for termination analysis of logic programs. TPLP. To appear.

Schneider-Kamp, P., Giesl, J., Serebrenik, A., and Thiemann, R. 2009. Automated termination proofs for logic programs by term rewriting. ACM TOCL 11, 1.

Schneider-Kamp, P., Giesl, J., Serebrenik, A., Ströder, T., And Thiemann, R. 2010. Automated termination analysis for logic programs with cut. Tech. Rep. AIB2010-10, RWTH Aachen. http://aib.informatik.rwth-aachen.de 
Serebrenik, A. AND De Schreye, D. 2005. On termination of meta-programs. TPLP 5, 3, 355-390.

Sørensen, M. H. AND GLÜCK, R. 1995. An algorithm of generalization in positive supercompilation. In ILPS '95. MIT Press, 465-479.

Spoto, F. And Levi, G. 1998. Abstract interpretation of Prolog programs. In AMAST '98. LNCS 1548. 455-470.

Spoto, F. 2000. Operational and goal-independent denotational semantics for Prolog with cut. J. Log. Prog. 42, 1, 1-46.

STRÖDER, T. 2010. Towards termination analysis of real Prolog programs. Diploma Thesis, RWTH Aachen. http://aprove.informatik.rwth-aachen.de/eval/Cut/ 